\title{
Direct two-dimensional measurements of the field-aligned current associated with plasma blobs
}

\author{
I. Furno ${ }^{1}$, M. Spolaore ${ }^{2}$, C. Theiler ${ }^{1}$, N. Vianello ${ }^{2}$, R. Cavazzana $^{2}$, and A. Fasoli ${ }^{1}$ \\ ${ }^{1}$ Centre de Recherches en Physique des Plasmas-Ecole Polytechnique Fédérale de Lausanne, \\ Association EURATOM-Confédération Suisse, CH-1015 Lausanne, Switzerland \\ ${ }^{2}$ Consorzio RFX, Associazione Euratom-ENEA sulla Fusione, Corso Stati Uniti 4, 35127 Padova, Italy
}

(Dated: January 17, 2011)

\begin{abstract}
In simple magnetized toroidal plasmas, field-aligned blobs originate from ideal interchange waves and propagate radially outward under the effect of $\nabla B$ and curvature induced $\mathbf{E} \times \mathbf{B}$ drifts. We report on first experimental two-dimensional measurements of the field-aligned current associated with blobs, whose ends terminate on a conducting limiter. A dipolar structure of the current density is measured, which originates from $\nabla B$ and curvature induced polarization of the blob and is consistent with sheath boundary conditions. The dipole is strongly asymmetric due to the nonlinear dependence of the current density at the sheath edge upon the floating potential. Furthermore, we directly demonstrate the existence of two regimes, in which parallel currents to the sheath do or do not significantly damp charge separation and thus blob radial velocity.
\end{abstract}

PACS numbers: 52.35.Ra, 52.35.Kt, 52.25.Fi

Filamentary current structures associated with intermittent blobs and filaments, which propagate across magnetic fields, are observed in natural and laboratory plasmas. For example, satellites reveal current filaments aligned with the Earth magnetic field [1], which are transported with ionospheric blobs. On RFX, a reversed field pinch, insertable magnetic probes measured field aligned currents associated with plasma blobs [2]. In tokamaks, current structures associated with plasma filaments during edge localized mode (ELMs) were measured using fast imaging combined with external magnetic data on MAST [3], reciprocating magnetic probe data in the Scrape Off Layer (SOL) of ASDEX Upgrade [4] and JET tokamaks [5], and filament modeling and comparison with external coil data on DIII-D [6]. Although the field-aligned nature of these current filaments is a common feature, detailed measurements of the two-dimensional (2D) structure in the plane perpendicular to the confining field are missing, thus preventing a fuller understanding of the origin of the current itself. Furthermore, to date, the importance of parallel currents on blob propagation is only indirectly inferred by comparing experimental blob size-versus-speed scalings $[7,8]$ with theory predictions [9].

In this Letter, we report on first two-dimensional measurements of the parallel current density associated with radially propagating blobs in the open field line geometry of a simple magnetized toroidal plasma. The experiments are performed on the TORPEX device [10]. Blobs are generated from ideal interchange waves [11] and are propelled by the effective gravity force associated with $\nabla B$ and magnetic field curvature causing charge separation, polarizing the blob and a corresponding radially outwards $\mathbf{E} \times \mathbf{B}$ drift $[7,12]$. Blobs propagate in a region where both of their ends are connected to a conducting limiter, where a plasma sheath is formed. Time-resolved $2 \mathrm{D}$ profiles of parallel current density to the limiter are obtained using data from a single-sided Langmuir probe (LP) and from a specially designed current probe, based on an array of magnetic pick-up coils, which are conditionally sampled over many blob events. A dipolar structure of the parallel current density is revealed, which originates from blob-induced charge separation. This dipolar structure is consistent with sheath boundary conditions and is strongly asymmetric, resulting in a net current to the limiter. Furthermore, by using these internal measurements, we directly demonstrate the existence of two regimes, in which parallel currents to the sheath do or do not significantly damp charge separation, and thus blob radial velocity, as concluded in Ref. [7] from a comparison of experimental measurements with a blob speedversus-size scaling law, predicted by theory.

TORPEX (major radius $R=1 \mathrm{~m}$, minor radius $a=0.2$ $\mathrm{m})$ plasmas are produced and sustained by microwaves $\left(P_{E C} \approx 400 \mathrm{~W}\right)$ in the electron cyclotron range of frequencies [13]. A vertical magnetic field $B_{z}=1.6$ $\mathrm{mT}$ is imposed on a toroidal field of $B_{t}=76 \mathrm{mT}$, resulting in helicoidal magnetic field lines with a $\nabla B$ and curvature, that terminate on the lower and upper walls of the vessel. In this simple magnetized configuration, ideal interchange modes with parallel, $k_{\|}=0$, and perpendicular, $k_{z}=2 \pi / \Delta$, wave numbers dominate over resistive interchange modes [14], provided that $2 \pi N \rho_{s} c_{s} /\left(0.3 L_{v} R \gamma_{I}\right) \lesssim 1$. Here, $\rho_{s}=c_{s} / \Omega_{i}, c_{s}=$ $\sqrt{T_{e} / m_{i}}, \Omega_{i}=e B_{0} / m_{i}, \gamma_{I} \sim \sqrt{2} c_{s} / \sqrt{R L_{p}}$ is the ideal interchange linear growth rate, $N=L_{v} / \Delta$ is the number of turns of a field line from top to bottom of the device, $\Delta=2 \pi R B_{z} / B_{t}, L_{v}$ is the height of the vacuum vessel and $L_{p}$ is the electron pressure scale length. This condition is satisfied here $\left(\rho_{s} \sim 4 \mathrm{~mm}, L_{p} \sim 5 \mathrm{~cm}\right.$, and $N \approx 3$ ), and measurements [15] confirm that the present plasmas fall in the regime dominated by an ideal interchange instability that develops around the position of 


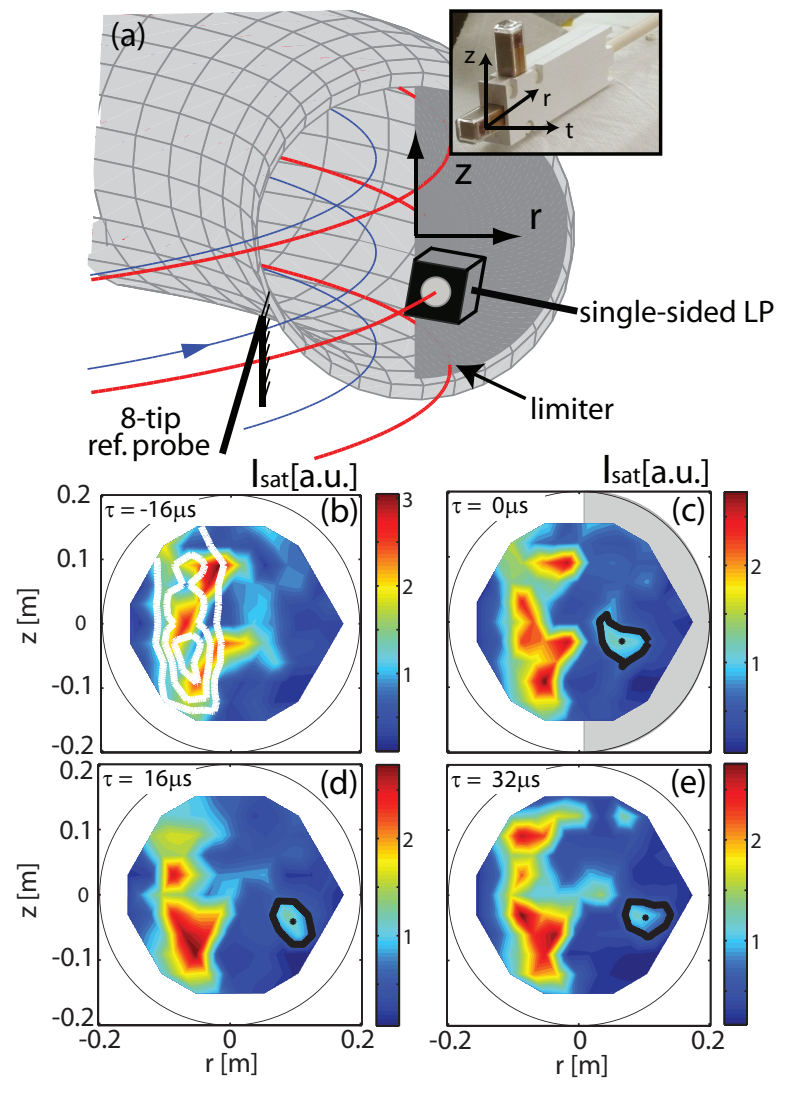

FIG. 1: (a) Schematics (not to scale) of the setup with examples of magnetic field lines. The one in violet does $\approx 3$ turns before intercepting the vessel walls; the one in red lies in the blob propagation region and intercepts the limiter plate after one turn. Either the single-sided LP or the current probe (see picture in the inset with the relevant coordinate system) is placed in front of the limiter. The eight-tip reference probe is toroidally displaced by $\approx 3 \mathrm{~m}$ from the limiter. (b-e) $2 \mathrm{D}$ profiles of ion saturation current from a $2 \mathrm{D}$ LP array, which is toroidally spaced by $\approx 90$ degrees from the limiter. An example of blob is highlighted by the thick black line.

minimum $L_{p}$ at $r \approx-3 \mathrm{~cm}$.

Similarly to the scenario extensively studied in Refs. $[7,11,16]$, blobs form from radially extending positive crests of the ideal interchange wave that are sheared off by the $\mathbf{E} \times \mathbf{B}$ flow. The radial elongation of the wave is attributed to a decrease in the radial pressure scale length $[11,17]$. A blob example is shown in Fig. 1, using $2 \mathrm{D}$ profiles of ion saturation current from a $2 \mathrm{D}$ LP array [18]. The elongation of the wave crest is shown in Fig. 1 (b), while the radial propagation of the newly formed blob is clearly visible in Fig. 1(c-e). For the present experiments, a steel limiter, schematically shown in Fig. $1(\mathrm{a})$, is inserted in the outer half of the cross section, i.e. $r>0$, where blobs propagate. This defines a region where blobs are connected on both sides of the limiter and have a nearly constant connection length $L_{\|}=2 \pi R \approx 6$ $\mathrm{m}$ [7]. The near perpendicular incidence of magnetic field lines to the limiter avoids complicating effects such as the contribution of the electron diamagnetic current to the blob parallel current, expected for small incidence angles [19].

In situ measurements of the parallel current density $J_{\|}$are a challenging task in thermonuclear oriented plasmas and various attempts have been done and are now in progress $[2,4,20]$. Here, $J_{\|}$is measured using two different diagnostics: a single-sided LP, and a specially designed current probe. The first probe consists of a tungsten plate $(8 \mathrm{~mm}$ in diameter, collecting area $A \approx 50$ $\mathrm{mm}^{2}$ ) with a single side exposed to the plasma. A schematical view of this probe is shown in Fig. 1. The plate is oriented perpendicularly to the magnetic field lines and is kept at the limiter potential, such that the current, $I_{0}$, flowing to the limiter is now measured by the probe. The current density is then computed as $J_{\|}=I_{0} / A$. The second probe consists of an L-shaped array of 3 miniaturized three-axial pick up coils $(3.5 \mathrm{~cm}$ spaced, each with an effective area of $\left.2.3 \times 10^{-3} \mathrm{~m}^{2}\right)$. This arrangement is a simplified version of that used in the Cluster satellite mission to measure magnetospheric currents [21], and allows direct measurements of $\mathbf{J}=\mu_{0}^{-1} \nabla \times \mathbf{B}$. The current probe is oriented such that the $(r, z)$ components of the magnetic field and their respective gradients along $r$ and $z$ are measured and the parallel current can be computed (see inset in Fig. 1). The signals are digitized at $250 \mathrm{kHz}$.

Time-resolved 2D profiles of $J_{\|}$are obtained by performing conditional sampling [22] over many blob events of the data in a time window centered around the blob detection. As schematically shown in Fig. 1, a multi-tip probe ( 8 tips, $1.8 \mathrm{~cm}$ spacing in the $z$ direction), located at $r=7 \mathrm{~cm}$ and toroidally displaced by $\approx 3 \mathrm{~m}$ from the limiter, is used as a reference probe. This measures ion saturation signals, $I_{\text {ref }}$, which are used to detect blobs on the different tips, defined by the condition $\widetilde{I}_{r e f} \geq 3 \sigma$, where $\widetilde{I}_{r e f}=I_{r e f}-\bar{I}_{\text {ref }}$ is the fluctuating signal and $\sigma$ is its standard deviation over the whole discharge. Two sets of measurements are performed, in which either the single-sided LP or the current probe is positioned $\approx 3$ $\mathrm{cm}$ in front of the limiter and is moved radially in between discharges, thus allowing reconstructing $J_{\|}$over a section of the $r-z$ poloidal plane. Furthermore, 2D profiles of electron density $n_{e}$, temperature $T_{e}$, and floating potential $V_{f l}$ are measured in the same poloidal plane and in the bulk plasma at $1 \mathrm{~m}$ distance from the limiter, by using conditionally sampled $\mathrm{I}-\mathrm{V}$ characteristics of a LP, which is moved between discharges. This technique is described in detail in Ref. [23].

Figure 2 illustrates the results of the conditional sampling of $\approx 3000$ blobs over four identical, 1 s long plasma discharges in hydrogen. At the blob detection time, the conditionally sampled $2 \mathrm{D}$ current density in a $r-z$ plane located $3 \mathrm{~cm}$ in front of the limiter is shown from single- 

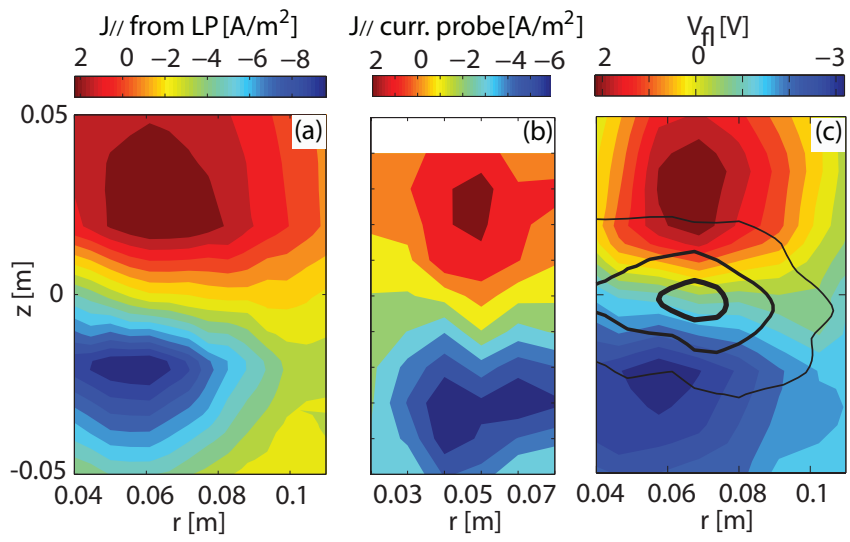

FIG. 2: 2D parallel current at the time of the blob detection measured $\sim 3 \mathrm{~cm}$ in front of the limiter by (a) single-sided LP and (b) current probe. Positive/negative currents correspond to excess of ions/electrons collected by the limiter. (c) $2 \mathrm{D}$ floating potential in the same plane together with iso-contours of blob density in the bulk plasma (1 m away from the limiter).

sided LP data, Fig. 2(a) and from current probe data, Fig. 2(b). The measurements from the two diagnostics are in excellent agreement. In the same plane, the conditionally sampled 2D structure of the floating potential is shown in Fig. 2(c), together with the density blob (shown by the black contours) as detected in the bulk plasma, i.e. $\approx 1 \mathrm{~m}$ away from the limiter. The density blob is mapped onto the first plane by taking into account the vertical shift of the field line $(\approx 2 \mathrm{~cm})$ over the distance between the two planes. The electron density and temperature at the center of the blob are respectively $n_{e} \approx 1.4 \times 10^{16} \mathrm{~m}^{-3}$ and $T_{e} \approx 2.5 \mathrm{eV}$. The floating potential in Fig. 2(c) exhibits an almost perfectly symmetric dipolar structure, centered around the density blob, with $\left|V_{f l}\right| \approx 3 \mathrm{~V}$ at the positions of minimum and maximum values. Surprisingly, the current density dipolar structure is not symmetric, with a larger level $\left(J^{-} \approx-9\right.$ $\mathrm{A} / \mathrm{m}^{-2}$ at the minimum) of current flowing out of the limiter on the bottom of the blob than that $\left(J^{+} \approx 2 \mathrm{~A} / \mathrm{m}^{-2}\right.$ at the maximum) flowing into the limiter at the top of the blob. To understand this asymmetry, we display in Fig. 3 the 2D parallel current density at the sheath entrance as computed from (a) $J_{\|}=J_{s a t}\left[1-\exp \left(-e V_{f l} / T_{e}\right)\right]$ [24], where $J_{\text {sat }}=0.5 \times n_{e} e c_{s}$ is the ion saturation current density, and (b) from the linearized expression $\left.J_{\|} \approx J_{s a t}\left[e V_{f l} / T_{e}\right)\right]$. The latter is often used as a closure scheme in theory and numerical simulations of SOL dynamics under the assumption that $\left|e V_{f l} / T_{e}\right| \ll 1[25]$. While the exact expression in Fig. 3 (a) is in excellent agreement with Fig. 2 (a-b), displaying an asymmetric dipolar structure, the linearized version in Fig. 3 (b) is almost perfectly symmetric. This is due to the assumption $\left|e V_{f l} / T_{e}\right| \ll 1$, which is not satisfied here $\left(\left|e V_{f l} / T_{e}\right| \approx 1\right)$, such that the linearized expression leads to large errors in the $J_{\|}$estimate. The asymmetry of the dipolar current
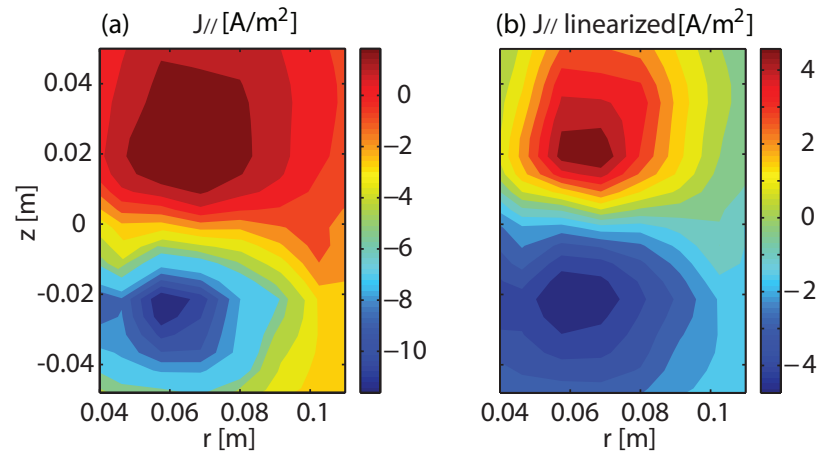

FIG. 3: Parallel current density (a) from sheath boundary condition $J_{\|}=J_{s a t}\left[1-\exp \left(-e V_{f l} / T_{e}\right)\right]$, and (b) from the linearized expression $\left.J_{\|} \approx J_{\text {sat }}\left[e V_{f l} / T_{e}\right)\right]$.

density can be quantified by the ratio $\alpha=\left|J^{-} / J^{+}\right|=$ $\left|\left[1-\exp \left(-e V_{f l}^{\min } / T_{e}\right)\right] /\left[1-\exp \left(-e V_{f l}^{\max } / T_{e}\right)\right]\right|$, where $J^{+}$ and $J^{-}$are the peak current densities in the positive and negative lobes respectively. For $\left|e V_{f l} / T_{e}\right| \approx 1$, we obtain $\left|J^{-} / J^{+}\right| \approx 2.7$, thus resulting in a net current to the limiter, which is carried by the electrons and exceeds in absolute value the ion saturation current.

Next, we address the key question of the role of the parallel current in damping charge separation, and thus in determining the blob radial speed. In TORPEX, it was recently demonstrated that a statistical blob speedversus-size scaling law, obtained by varying the ion mass, is consistent with the existence of two regimes, in which the blob speed is either limited by parallel current to the sheath or by ion polarization current [7]. Here, these two regimes, obtained for hydrogen and helium plasmas respectively, are investigated with internal measurements.

Using a simple 2D model for the blob (i.e. $k_{\|}=0$ ) $[25,26]$, neglecting ion temperature $\left(T_{i} \ll T_{e}\right.$ for TORPEX plasmas), and integrating $\nabla \cdot \mathbf{J}=0$ along the magnetic field, we obtain the parallel current to the sheath required, in the absence of other closure paths for the current, to completely damp the charge separation,

$$
\left.J_{\|}\right|_{\text {sheath }}=-\left.\frac{L_{\|}}{R B} \frac{\partial\left(n_{e} T_{e}\right)}{\partial z}\right|_{L / 2} .
$$

The r.h.s. of Eq. (1) represents the drive for blob motion and is evaluated on a vertical cut across the density blob center from LP measurements in the bulk plasma $(1 \mathrm{~m}$ away from the limiter) for hydrogen and helium blobs. The profiles are compared in Fig. 4 with the experimental profiles of the current density to the sheath. For hydrogen, Fig. 4(a), the profiles are of the same order of magnitude and agree within the errorbars for $z<0$. This is not the case for helium, Fig. 4(b), where the current density profile represents only a small fraction of the drive. We therefore conclude that parallel currents to the sheath damp a significant fraction of the charge separation in hydrogen, but not in helium, where other 

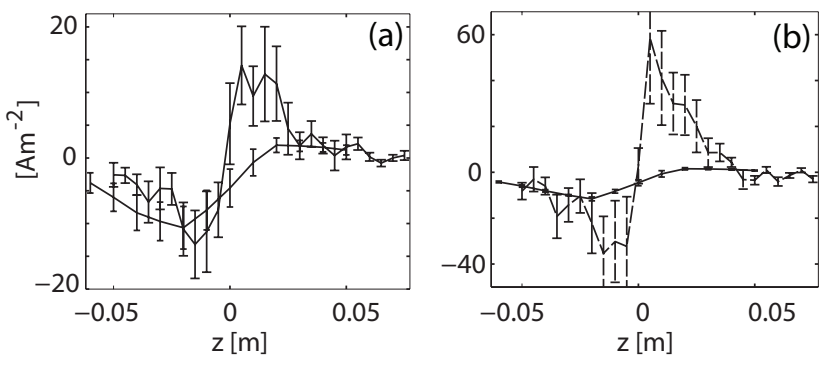

FIG. 4: Comparison of the measured parallel current density to the sheath (solid line) with the profile from Eq. (1) (dotted line) reveals that the former is effective in damping charge separation in hydrogen (a) but not in helium (b) blobs.

closure mechanisms, such as ion polarization current and ion current due to neutral friction, must be effective, in agreement with previous results [7].

The importance of the present results, in particular for fusion, is further amplified by similarities between blobs and ELM filaments, which suggest that the same mechanism is underlying the behavior of ELM filamentary substructures and blobs [27]. Recent measurements show the existence of parallel currents associated with filaments during ELMs [4], which result in large net currents to divertor plates [28]. These currents strongly perturb magnetic probe data, thus affecting machine operation, and may cause important changes in the magnetic field topology [29]. The origin and detailed spatial structure of these currents (monopolar [3, 30] versus dipolar [31] ) are a matter of debate. It is therefore interesting for ELM filaments to discuss the importance of the parallel current caused by the polarization of the filament and its asymmetric structure. An estimate of the net filament current (assuming a circular filament of radius $r$ ) is given by $I \approx(\alpha-1) J^{+} \times r^{2} \pi$, where the exact value of $\alpha$ (in our case $\alpha \approx 2.7$ ) depends upon the ratio $e V_{f l} / T_{e}$. In the SOL of tokamaks, typical filament parameters are $c_{s} \sim 30-60 \mathrm{~km} / \mathrm{s}, n_{e} \sim 1-3 \times 10^{19} \mathrm{~m}^{-3}$ and $r \sim 1-3$ $\mathrm{cm}$. Net currents in the range $\approx 50-500$ A should, thus, be expected, which are of the order of experimentally measured filament currents [32].

In summary, we presented first experimental 2D measurements of profiles of parallel current associated with plasma blobs in a simple, magnetized toroidal device. 2D dipolar structures of both floating potential and current density are measured. While the floating potential is almost a perfectly symmetric dipole, this is not the case for the current density, which exhibits a stronger lobe on the side dominated by electron currents. This is due to the non-linear dependence of the total current upon the floating potential. The relevance of the parallel current density asymmetry to ELM filaments was also discussed. Using internal measurements, we showed the existence of two regimes for blob propagation, obtained for hydrogen and helium plasmas, in which parallel current to the sheath respectively do or do not efficiently damp the $\nabla B$ and curvature induced polarization of the blob.

We thank insightful discussions with B.P. Duval, B. Labit, and P. Ricci. This work is partly supported by the Fonds National Suisse de la Recherche Scientifique and by the European Communities, under the contract of Association between EURATOM/ENEA, within the framework the European Fusion Development Agreement.

[1] J. Park et al., Annales Geophysicae 28, 697 (2010).

[2] M. Spolaore et al., Phys. Rev. Lett. 102, 165001 (2009).

[3] A. Kirk et al., Phys. Rev. Lett. 96, 185001 (2006).

[4] N. Vianello et al., Direct observation of current in type I ELM filaments on Asdex Upgrade, submitted to Phys. Rev. Lett.

[5] C. Silva et al., Plasma Phys. Controll. Fusion 51, 105001 (2009).

[6] H. Takahashi, E. Fredrickson, and M. Shaffer, Phys. Rev. Lett. 100, 205001 (2008).

[7] C. Theiler et al., Phys. Rev. Lett. 103, 065001 (2009).

[8] N. Katz et al., Phys. Rev. Lett. 101, 015003 (2008).

[9] S. Krasheninnikov, Phys. Lett. A 283, 368 (2001); O. E. Garcia et al., Phys. Plasmas 12, 090701 (2005); J. Myra and D. D'Ippolito, Phys. Plasmas 12, 092511 (2005).

[10] A. Fasoli et al., Plasma Phys. Contr. Fusion 52, 124020 (2010)

[11] I. Furno et al., Phys. Rev. Lett. 100, 055004 (2008).

[12] M. Podestà et al., Phys. Rev. Lett. 101, 045001 (2008).

[13] M. Podestà et al., Plasma Phys. Control. Fusion 47, 1989 (2005).

[14] P. Ricci and B. N. Rogers, Phys. Rev. Lett. 104, 145001 (2010).

[15] P. Ricci et al., Phys. Plasmas 16, 055703 (2009).

[16] S. H. Müller et al., Phys. Plasmas 14, 110704 (2008).

[17] C. Theiler et al., Phys. Plasmas 15, 042303 (2008).

[18] S. H. Müller et al., Phys. Plasmas 13, 100701 (2006).

[19] R. H. Cohen and D. D. Ryutov, Phys. Plasmas 12, 2011 (1995).

[20] M. F. M. De Bock et al., in Proceedings 37th EPS Conference on Plasma Physics, Dublin (2010), O2.107.

[21] J. V. Allen, Am. J. Phys. 74, 809 (2006).

[22] H. Johnsen, H. Pecseli, and J. Trulsen, Physics of Fluids 30, 2239 (1987).

[23] I. Furno et al., Phys. Plasmas 15, 055903 (2008).

[24] P. Stangeby, The Plasma Boundary of Magnetic Fusion Devices (Inst. of Phys. Pub. ISBN 075030559 2, 2000).

[25] S. I. Krasheninnikov et al., J. Plasma Phys. 74, 679 (2008).

[26] D. A. D'Ippolito et al., Phys. Plasmas 9, 222 (2002).

[27] D. L. Rudakov et al., Nucl. Fusion 45, 1589 (2005); E. Endler et al., Plasma Phys. Control. Fusion 47, 219 (2005); W. Fundamenski et al., Plasma Phys. Control. Fusion 49, R43 (2007).

[28] J. Lingertat et al., J. Nucl. mat. 402, 241 (1997); R. Pitts et al., Nucl. Fusion 43, 1145 (2003); T. Eich et al., J. Nucl. Mater. 669, 337 (2005).

[29] A. Wingen et al., Phys. Rev. Lett. 104, 175001 (2010).

[30] J. R. Myra, Phys. Plasmas 14, 102314 (2007).

[31] V. Rozhansky and A. Kirk, Plasma Phys. Control. Fusion 
50, 025008 (2008).

[32] P Migliucci et al., Phys. Plasmas 17, 072507 (2010);

(2006).

A. Kirk et al., Plasma Phys. Control. Fusion 48, B433 